\title{
Riqueza de dipterofauna associada à matéria orgânica em decomposição em fragmentos de Floresta Estacional Semidecidual no sul de Goiás
}

\author{
José Eduardo Neto de Sousa ${ }^{1,2 *}$ \\ Lucas Silva de Faria ${ }^{2}$ \\ Julio Mendes ${ }^{2}$ \\ Narcisa Silva Soares ${ }^{1}$ \\ ${ }^{1}$ Instituto Luterano de Ensino Superior de Itumbiara - ILES/ULBRA \\ Avenida Beira Rio, 1001, Nova Aurora, CEP 75522-330, Itumbiara - GO, Brasil \\ ${ }^{2}$ Universidade Federal de Uberlândia, Campus Umuarama, Uberlândia - MG, Brasil \\ * Autor para correspondência \\ jnetodesousa@yahoo.com.br
}

Submetido em 14/03/2014

Aceito para publicação em 12/08/2014

\section{Resumo}

A diversidade de Diptera foi estudada em quatro fragmentos de floresta estacional semidecidual no sul de Goiás. A riqueza das famílias Muscidae, Calliphoridae e Sarcophagidae foi analisada. Os insetos foram coletados bimestralmente durante um ano por meio de armadilhas com iscas em decomposição. As armadilhas foram mantidas durante cinco dias nos fragmentos. Um total de 1.956 indivíduos pertencentes a 13 famílias foram coletados. Foram identificadas 29 espécies pertencentes às principais famílias. Muscidae mostrou maior abundância enquanto Sarcophagidae apresentou maior número de espécies. Houve um novo registro da espécie Philornis schildi Dodge, 1963 (Muscidae) no território brasileiro. Estes dados reforçam a importância da preservação dessas áreas e novos estudos para melhorar o conhecimento da biodiversidade associada a estes fragmentos. Esta informação também pode ser útil em futuros estudos sobre o impacto da ação humana sobre a fauna de insetos associados a este ambiente.

Palavras-chave: Cerrado; Diptera; Itumbiara-GO; Philornis schildi

\section{Abstract}

Dipteran fauna associated with decaying organic matter in semi-deciduous forest fragments in southern Goiás, Brazil. Dipteran diversity was studied in four seasonal, semi-deciduous forest fragments in southern Goiás state, Brazil. Species richness of Muscidae, Calliphoridae and Sarcophagidae families was analyzed. The insects were collected each two months over the course of one year, using traps with decaying bait. The traps were kept in the field for five days each sampling period. A total of 1,956 individuals belonging to 13 families were collected. 29 species were identified belonging to the main families. Muscidae had the highest abundance, while Sarcophagidae presented the greatest number of species. We also report the occurrence of Philornis schildi (Muscidae) for the first time in Brazil. These data reinforce the importance of preserving these areas, and indicate the need for new studies to improve knowledge of the biodiversity associated with these fragments. This information may also be useful in future studies regarding the impact of human activity on insect fauna associated with this environment.

Key words: Cerrado; Diptera; Itumbiara-GO; Philornis schildi 


\section{Introdução}

Com o crescimento da população humana, os ecossistemas naturais estão cada vez mais expostos a múltiplas pressões antrópicas, principalmente pela intensificação da urbanização, agricultura e pecuária. São fatores que induzem ao desmatamento de vastas áreas e que, consequentemente, levam à fragmentação de ambientes naturais (BARBOLA et al., 2007; MCLAUGHLIN et al., 2013). Este evento pode afetar significativamente o sucesso reprodutivo das plantas (NEWMAN et al., 2013) e ocasionar o isolamento de populações ou a extinção de determinadas espécies (BARBOLA et al., 2007). A fragmentação também favorece a invasão de espécies exóticas, erosão dos solos, poluição de aquíferos, degradação de ecossistemas, alterações nos regimes de queimadas, desequilíbrios no ciclo do carbono e possivelmente modificações climáticas regionais (KLINK; MACHADO, 2005; JACKSON; FAHRIG, 2013).

O Cerrado brasileiro é um dos biomas com maior necessidade de conservação a nível mundial (MYERS et al., 2000). Destaca-se por apresentar grande extensão territorial em uma região marcada pela expansão da ocupação humana, associada a uma grande biodiversidade ameaçada e ainda pouco estudada (RATTER et al., 1997; BRASIL, 2002). Diante desta ameaça, tem aumentado o número de trabalhos realizados para avaliar o impacto da crescente fragmentação das áreas naturais em relação à biodiversidade. Neste contexto, a entomofauna assume um papel de extrema importância pela abundância na natureza, riqueza de espécies e a participação em diversos processos ecológicos (GULLAN; CRANSTON, 2008).

Dentre os insetos, a ordem Diptera destaca-se como a quarta ordem em número de espécies, possui distribuição cosmopolita, tem como fonte de alimento uma grande variedade de substratos e está associada tanto a áreas naturais quanto a áreas modificadas pela ação humana. São importantes também na dinâmica dos ecossistemas, atuando como polinizadores e em processos de ciclagem de nutrientes como decompositores (MARICONI et al., 1998). Além disso, possuem importância médica e veterinária, pela veiculação e transmissão de agentes etiológicos, importância agrícola, por serem pragas de diversas culturas, e, mais recentemente, têm sido utilizados em investigações criminais e no tratamento de feridas crônicas por meio da terapia larval (MORALES; KÖHLER, 2006; OLIVEIRA et al., 2008).

O conhecimento da fauna e flora em ecossistemas pouco estudados é importante para prevenções ou remediações de impactos nos diferentes ambientes. Além disso, estudos desta natureza podem identificar potenciais grupos/espécies indicadores ecológicos (THOMANZINI; THOMANZINI, 2002; WINK et al., 2005; AZEVEDO et al., 2011). Ainda são escassos estudos sobre a caracterização da dipterofauna em remanescentes de Floresta Estacional Semidecidual inseridos no bioma Cerrado e particularmente no sul de Goiás, onde tem havido desflorestamento desse perfil vegetacional (SOS MATA ATLÂNTICA; INPE, 2011).

Nesse contexto, o reconhecimento da biodiversidade tem estimulado a criação de diversos tipos de inventários. Assim, quanto maior for o conhecimento sobre os ecossistemas, maior será a possibilidade de explorá-los adequadamente, com menor dano e maior longevidade de seus componentes. A proteção do solo e das espécies que ele abriga significará, numa visão mais ampla, maior bem-estar para a espécie humana (MELO, 2008).

O objetivo deste estudo foi caracterizar quatro fragmentos de Floresta Estacional Semidecidual do sul de Goiás quanto à diversidade de dípteros e verificar a riqueza e abundância das famílias: Muscidae, Calliphoridae e Sarcophagidae.

\section{Material e Métodos}

Os experimentos foram realizados em quatro fragmentos (F1, F2, F3 e F4) de Floresta Estacional Semidecidual, localizados a aproximadamente $14 \mathrm{~km}$ do município de Itumbiara e a $20 \mathrm{~km}$ do município de Buriti Alegre, ambos pertencentes à região sul do estado de Goiás, Brasil. Todos os fragmentos estudados são áreas de Reservas Legais de fazendas particulares, formados por sub-bosques densos e ralos, marcados pela presença de arvoretas e arbustos com altura média variando entre 1,8 m e 3,5 m, e cercados por áreas destinadas a práticas agropastoris. As distâncias entre os fragmentos variam de cerca de $700 \mathrm{~m}$ a $6 \mathrm{~km}$. F1 apresenta extensão territorial de 57 ha (18 $\left.20^{\prime} 57.85^{\prime \prime} \mathrm{S}-4^{\circ} 4^{\prime} 12.61^{\prime \prime O}\right), \mathrm{F} 2$ abrange

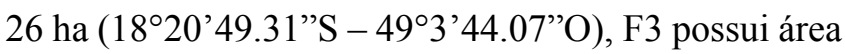


de 34 ha $\left(18^{\circ} 17^{\prime} 43.41\right.$ 'S $\left.-49^{\circ} 5^{\prime} 56.32^{\prime \prime O}\right)$ e F4 tem dimensão de 22 ha ( $18^{\circ} 18^{\prime} 35.45^{\prime \prime} \mathrm{S}-49^{\circ} 5^{\prime}$ '16.80”O). Os fragmentos foram selecionados como forma de repetição para caracterização do ambiente (Figura 1).

As coletas dos insetos foram realizadas bimestralmente, durante o período de um ano, totalizando seis coletas. Em cada fragmento, foi estabelecido um transecto paralelo à borda de maior extensão, distante 60 $\mathrm{m}$ da mesma, onde foram instaladas seis armadilhas aéreas para insetos voadores, distantes $50 \mathrm{~m}$ entre si (Figura 2). As armadilhas foram construídas de acordo com Lomônoco e Almeida (1995) e Marchiori et al. (2003c) utilizando recipientes do tipo garrafa PET com iscas colocadas na base inferior de cada armadilha (três com fezes humanas e três com moela de frango) e fixadas a uma altura de 1,20 m(LOMÔNOCO; ALMEIDA, 1995). Após a instalação, as armadilhas foram mantidas em campo por cinco dias. Em seguida, foram retiradas e levadas ao laboratório para triagem. Os espécimes coletados foram conservados em etanol $70 \%$ e posteriormente identificados. Os insetos coletados foram identificados com o auxílio de chaves taxonômicas (McALPINE, 1987; CARVALHO et al., 2002; CARVALHO; MELLO-PATIU, 2008; VAIRO et al., 2011), por meio de comparações com os exemplares da coleção entomológica do Laboratório de Entomologia Médica e Veterinária do setor de Parasitologia, da Universidade Federal de Uberlândia/UFU e com a contribuição da pesquisadora do Museu Nacional da Universidade Federal do Rio de Janeiro, Dr ${ }^{\mathrm{a}}$ Márcia Souto Couri, especialista em taxonomia de Muscidae. Optou-se por agrupar as amostras coletadas nos dois tipos de iscas para analise das frequências das famílias e espécies nos vários fragmentos de mata amostrados.

As frequências, absoluta e relativa, dos indivíduos em cada fragmento foram determinadas utilizando o programa computacional Excel, 2013 (Microsoft Corporation, Redmond, WA, EUA).

FIGURA 1: Fragmentos de Floresta Estacional Semidecidual em Itumbiara-GO utilizados no estudo. F1 = fragmento 1; F2 = fragmento 2; $\mathrm{F} 3=$ fragmento $3 ; \mathrm{F} 4=$ fragmento 4.

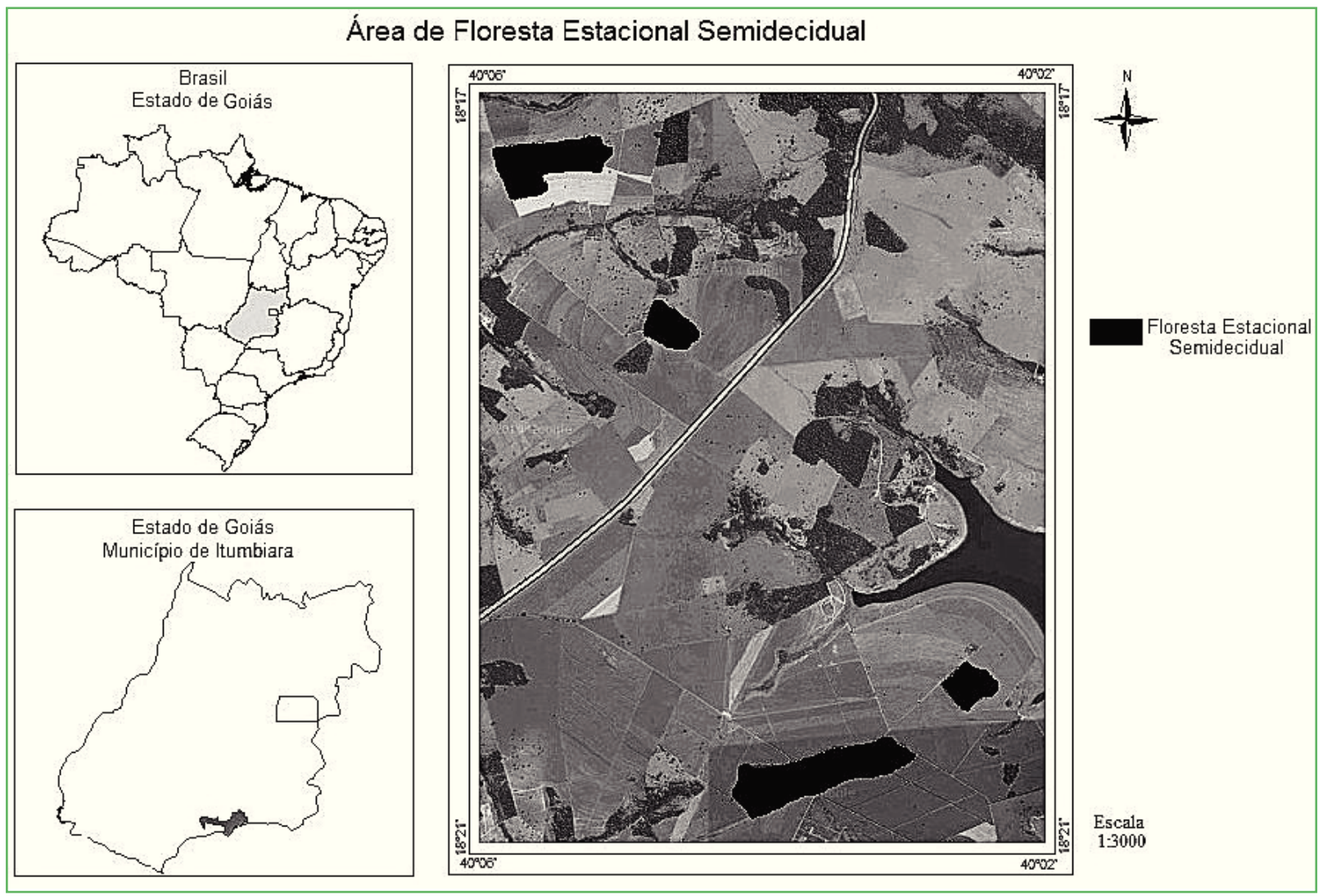


FIGURA 2: Desenho amostral realizado nos quatro fragmentos de Floresta Estacional Semidecidual em Itumbiara - GO. P $=$ pontos amostrados. P1, P3 e P5 = pontos amostrados com iscas de moelas de frango. P2, P4 e P6 = pontos amostrados com iscas de fezes humanas.

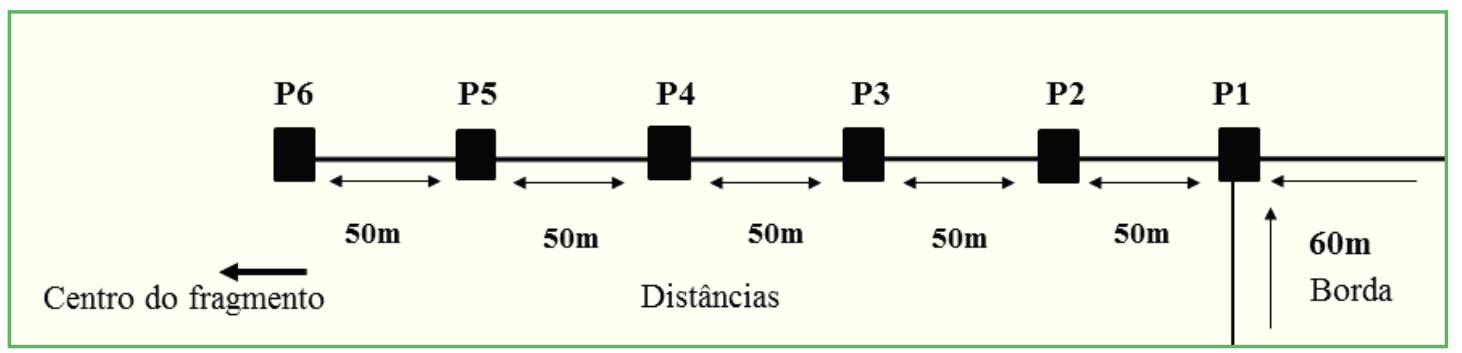

\section{Resultados}

Foram capturados 1.956 dípteros, pertencentes a 13 famílias. A família Muscidae apresentou a maior abundância nos quatro fragmentos com 555 (28,37\%) indivíduos coletados, seguida das famílias Phoridae (n $=368-18,81 \%)$, Sarcophagidae $(\mathrm{n}=302-15,44 \%)$, Ulidiidae $(\mathrm{n}=251-12,83 \%)$, Anthomyiidae $(\mathrm{n}=225$ $-11,50 \%$ ) e demais famílias com $n=255$ e $13,04 \%$ dos indivíduos (Tabela 1).

O fragmento 4 (F4) apresentou maior abundância com 873 indivíduos (45\%), seguido por F2 com 428 (22\%), F1 com 341 (18\%) e F3 com 278 (15\%) indivíduos cada. As famílias Muscidae e Anthomyiidae apresentaram maior abundância em F4 com 360 e 136 indivíduos, respectivamente; enquanto que, Phoridae e
Ulidiidae tiveram maior abundância em F3 com 102 e 55 indivíduos, respectivamente. A família Fanniidae foi exclusiva do F1, bem como Tabanidae em F3.

A família Sarcophagidae apresentou maior riqueza com 18 espécies, sendo o gênero Oxysarcodexia (Townsend, 1917) mais abundante, seguido de Peckia (Robineau-Desvoidy, 1830). A espécie Sarcodexia lambens (Wiedemann, 1830) foi a mais abundante com 90 indivíduos coletados (29,80\%), seguida por Peckia (Euboettcheria) collusor (Curran \& Walley, 1934) com 70 indivíduos $(23,18 \%)$, ambas presentes em todos os fragmentos estudados (Tabela 2). Helicobia aurescens (Townsend, 1927), Helicobia morionella Aldrich, 1930, Helicobia rapax (Walker, 1849) e Udamopyga percita (Lopes, 1938) ocorreram exclusivamente em F4.

TABELA 1: Frequência absoluta (FA) e relativa (FR) das famílias de dípteros coletados em quatro fragmentos (F1, F2, F3 e F4) de Floresta Estacional Semidecidual em Itumbiara, GO.

\begin{tabular}{|c|c|c|c|c|c|c|c|c|c|c|}
\hline \multirow{3}{*}{ Família } & \multicolumn{8}{|c|}{ Fragmentos } & \multirow{2}{*}{\multicolumn{2}{|c|}{ Total }} \\
\hline & \multicolumn{2}{|c|}{ F1 } & \multicolumn{2}{|c|}{ F2 } & \multicolumn{2}{|c|}{ F3 } & \multicolumn{2}{|c|}{ F4 } & & \\
\hline & $\mathrm{FA}$ & FR $(\%)$ & $\mathrm{FA}$ & FR $(\%)$ & FA & FR $(\%)$ & FA & FR $(\%)$ & FA & FR $(\%)$ \\
\hline Anthomyiidae & 26 & 7,62 & 63 & 14,72 & - & 0,00 & 136 & 15,58 & 225 & 11,50 \\
\hline Calliphoridae & 11 & 3,23 & 15 & 3,50 & 5 & 1,80 & 20 & 2,29 & 51 & 2,61 \\
\hline Drosophilidae & 47 & 13,78 & 21 & 4,91 & 5 & 1,80 & 42 & 4,81 & 115 & 5,88 \\
\hline Fanniidae & 48 & 14,08 & - & 0,00 & - & 0,00 & - & 0,00 & 48 & 2,45 \\
\hline Micropezidae & 9 & 2,64 & - & 0,00 & 5 & 1,80 & 1 & 0,11 & 15 & 0,77 \\
\hline Muscidae & 71 & 20,82 & 101 & 23,60 & 23 & 8,27 & 360 & 41,24 & 555 & 28,37 \\
\hline Phoridae & 65 & 19,06 & 112 & 26,17 & 102 & 36,69 & 89 & 10,19 & 368 & 18,81 \\
\hline Sarcophagidae & 49 & 14,37 & 72 & 16,82 & 68 & 24,46 & 113 & 12,94 & 302 & 15,44 \\
\hline Sciaridae & - & 0,00 & 1 & 0,23 & 1 & 0,36 & - & 0,00 & 2 & 0,10 \\
\hline Tabanidae & - & 0,00 & - & 0,00 & 4 & 1,44 & - & 0,00 & 4 & 0,20 \\
\hline Tachinidae & 2 & 0,59 & - & 0,00 & 3 & 1,08 & 1 & 0,11 & 6 & 0,31 \\
\hline Tephritidae & 2 & 0,59 & 2 & 0,47 & 7 & 2,52 & 3 & 0,34 & 14 & 0,72 \\
\hline Ulidiidae & 47 & 13,78 & 41 & 9,58 & 55 & 19,78 & 108 & 12,37 & 251 & 12,83 \\
\hline Total & 341 & 18 & 428 & 22 & 278 & 15 & 873 & 45 & 1956 & 100 \\
\hline
\end{tabular}


TABELA 2: Frequência absoluta (FA) e relativa (FR) das espécies de Calliphoridae, Muscidae e Sarcophagidae coletadas em quatro fragmentos de Floresta Estacional Semidecidual em Itumbiara, GO.

\begin{tabular}{|c|c|c|c|c|c|c|c|c|c|c|}
\hline \multirow{3}{*}{$\begin{array}{ll} & \text { Taxón } \\
\text { Calliphoridae } & \\
\end{array}$} & \multicolumn{8}{|c|}{ Fragmentos } & \multirow{2}{*}{\multicolumn{2}{|c|}{ Total }} \\
\hline & \multicolumn{2}{|r|}{ F1 } & \multicolumn{2}{|c|}{ F2 } & \multicolumn{2}{|c|}{ F3 } & \multicolumn{2}{|c|}{ F4 } & & \\
\hline & FA & FR $(\%)$ & FA & FR $(\%)$ & FA & FR $(\%)$ & FA & FR $(\%)$ & FA & FR $(\%)$ \\
\hline Chrysomya albiceps (Wiedemann 1819) & 6 & $54,55 \%$ & 5 & $33,33 \%$ & - & $0,00 \%$ & 6 & $30,00 \%$ & 17 & $33,33 \%$ \\
\hline Chrysomya megacephala (Fabricius, 1794) & - & $0,00 \%$ & 2 & $13,33 \%$ & - & $0,00 \%$ & - & $0,00 \%$ & 2 & $3,92 \%$ \\
\hline Chloroprocta idioidea (Robineau-Desvoidy, 1830) & - & $0,00 \%$ & 2 & $13,33 \%$ & 5 & $100,00 \%$ & 9 & $45,00 \%$ & 16 & $31,37 \%$ \\
\hline Hemilucilia segmentaria (Fabricius, 1805) & 2 & $18,18 \%$ & - & $0,00 \%$ & - & $0,00 \%$ & - & $0,00 \%$ & 2 & $3,92 \%$ \\
\hline Mesembrinella bicolor (Fabricius, 1805) & 3 & $27,27 \%$ & 4 & $26,67 \%$ & - & $0,00 \%$ & 3 & $15,00 \%$ & 10 & $19,61 \%$ \\
\hline Lucilia eximia (Wiedemann, 1819) & - & $0,00 \%$ & 2 & $13,33 \%$ & - & $0,00 \%$ & 2 & $10,00 \%$ & 4 & $7,84 \%$ \\
\hline Total Calliphoridae & 11 & $21,57 \%$ & 15 & $29,41 \%$ & 5 & $9,80 \%$ & 20 & $39,22 \%$ & 51 & $100,00 \%$ \\
\hline \multicolumn{11}{|l|}{ Muscidae } \\
\hline Atherigona orientalis (Schiner, 1868) & 44 & $61,97 \%$ & 72 & $71,29 \%$ & 12 & $52,17 \%$ & 159 & $44,17 \%$ & 287 & $51,71 \%$ \\
\hline Biopyrellia bipuncta (Wiedemann, 1830) & 9 & $12,68 \%$ & 7 & $6,93 \%$ & - & $0,00 \%$ & 14 & $3,89 \%$ & 30 & $5,41 \%$ \\
\hline Cyrtoneuropsis brunnea (Hough, 1900) & 15 & $21,13 \%$ & 5 & $4,95 \%$ & - & $0,00 \%$ & 51 & $14,17 \%$ & 71 & $12,79 \%$ \\
\hline Cyrtoneuropsis similata (Couri, 1982) & - & $0,00 \%$ & 15 & $14,85 \%$ & 6 & $26,09 \%$ & 128 & $35,56 \%$ & 149 & $26,85 \%$ \\
\hline Philornis schildi Dodge, 1963 & 3 & $0,00 \%$ & 2 & $1,98 \%$ & 5 & $21,74 \%$ & 8 & $2,22 \%$ & 18 & $3,24 \%$ \\
\hline Total Muscidae & 71 & $12,79 \%$ & 101 & $18,20 \%$ & 23 & $4,14 \%$ & 360 & $64,86 \%$ & 555 & $100,00 \%$ \\
\hline \multicolumn{11}{|l|}{ Sarcophagidae } \\
\hline Helicobia aurescens (Townsend, 1927) & - & $0,00 \%$ & - & $0,00 \%$ & - & $0,00 \%$ & 5 & $4,42 \%$ & 5 & $1,66 \%$ \\
\hline Helicobia morionella (Aldrich, 1930) & - & $0,00 \%$ & - & $0,00 \%$ & - & $0,00 \%$ & 3 & $2,65 \%$ & 3 & $0,99 \%$ \\
\hline Helicobia rapax (Walker, 1849) & - & $0,00 \%$ & - & $0,00 \%$ & - & $0,00 \%$ & 5 & $4,42 \%$ & 5 & $1,66 \%$ \\
\hline Oxysarcodexia avuncula (Lopes, 1933) & - & $0,00 \%$ & 1 & $1,39 \%$ & 1 & $1,47 \%$ & 2 & $1,77 \%$ & 4 & $1,32 \%$ \\
\hline Oxysarcodexia diana (Lopes, 1933) & 1 & $2,04 \%$ & - & $0,00 \%$ & 4 & $5,88 \%$ & 5 & $4,42 \%$ & 10 & $3,31 \%$ \\
\hline Oxyvinia excisa (Lopes, 1950) & - & $0,00 \%$ & - & $0,00 \%$ & 1 & $1,47 \%$ & 1 & $0,88 \%$ & 2 & $0,66 \%$ \\
\hline Oxysarcodexia major (Lopes, 1946) & - & $0,00 \%$ & - & $0,00 \%$ & 1 & $1,47 \%$ & 5 & $4,42 \%$ & 6 & $1,99 \%$ \\
\hline Oxysarcodexia paulistanensis (Mattos, 1919) & - & $0,00 \%$ & - & $0,00 \%$ & 2 & $2,94 \%$ & 2 & $1,77 \%$ & 4 & $1,32 \%$ \\
\hline Oxysarcodexia thornax (Walker 1849) & 9 & $18,37 \%$ & 13 & $18,06 \%$ & 4 & $5,88 \%$ & 20 & $17,70 \%$ & 46 & $15,23 \%$ \\
\hline Peckia (Euboettcheria) anguilla (Curran \& Walley, 1934) & 3 & $6,12 \%$ & - & $0,00 \%$ & 7 & $10,29 \%$ & 2 & $1,77 \%$ & 12 & $3,97 \%$ \\
\hline Peckia (Peckia) chrysostoma (Wiedemann, 1830) & 1 & $2,04 \%$ & 5 & $6,94 \%$ & - & $0,00 \%$ & - & $0,00 \%$ & 6 & $1,99 \%$ \\
\hline Peckia (Euboettcheria) collusor (Curran \& Walley, 1934) & 14 & $28,57 \%$ & 15 & $20,83 \%$ & 10 & $14,71 \%$ & 31 & $27,43 \%$ & 70 & $23,18 \%$ \\
\hline Peckia (Euboettcheria) florencioi (Prado \& Fonseca, 1932) & 4 & $8,16 \%$ & - & $0,00 \%$ & 2 & $2,94 \%$ & 3 & $2,65 \%$ & 9 & $22,50 \%$ \\
\hline Peckia (Squamatodes) ingens (Walker, 1849) & 1 & $2,04 \%$ & - & $0,00 \%$ & 4 & $5,88 \%$ & - & $0,00 \%$ & 5 & $1,66 \%$ \\
\hline Peckia (Pattonella) intermutans (Walker, 1861) & 2 & $4,08 \%$ & 5 & $6,94 \%$ & 6 & $8,82 \%$ & - & $0,00 \%$ & 13 & $4,30 \%$ \\
\hline Ravinia belforti (Prado \& Fonseca,1932) & 1 & $2,04 \%$ & 2 & $2,78 \%$ & - & $0,00 \%$ & 5 & $4,42 \%$ & 8 & $2,65 \%$ \\
\hline Sarcodexia lambens (Wiedemann, 1830) & 12 & $24,49 \%$ & 30 & $41,67 \%$ & 26 & $38,24 \%$ & 22 & $19,47 \%$ & 90 & $29,80 \%$ \\
\hline Udamopyga percita (Lopes, 1938) & - & $0,00 \%$ & - & $0,00 \%$ & - & $0,00 \%$ & 1 & $0,88 \%$ & 1 & $0,33 \%$ \\
\hline sp. 1 & 1 & $2,04 \%$ & - & $0,00 \%$ & - & $0,00 \%$ & - & $0,00 \%$ & 1 & $0,33 \%$ \\
\hline sp. 2 & - & $0,00 \%$ & - & $0,00 \%$ & - & $0,00 \%$ & 1 & $0,88 \%$ & 1 & $0,33 \%$ \\
\hline sp. 3 & - & $0,00 \%$ & 1 & $1,39 \%$ & - & $0,00 \%$ & - & $0,00 \%$ & 1 & $0,33 \%$ \\
\hline
\end{tabular}

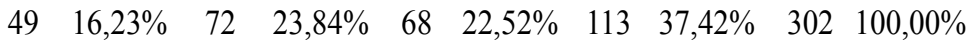


Na família Muscidae foram identificadas cinco espécies. Atherigona orientalis (Schiner, 1868) foi a espécie mais abundante e presente em todos os fragmentos com 287 indivíduos coletados (51\%). Houve um registro inédito da espécie Philornis shildi Dodge, 1963 no território brasileiro.

Calliphoridae apresentou seis espécies, sendo representada principalmente pela espécie Chrysomya albiceps (Wiedemann 1819) com 17 indivíduos coletados (33\%), ausentes apenas em F3. Chloroprocta idioidea (Robineau-Desvoidy, 1830) foi a segunda mais abundante, com 16 indivíduos (31,37\%) concentrados, principalmente em F4. Chrysomya megacephala (Fabricius, 1794) e Hemilucia segmentaria (Fabricius, 1805) apresentaram ocorrências restritas a um único fragmento, F2 e F1, respectivamente.

\section{Discussão}

As famílias de dípteros encontradas no presente estudo foram similares às da pesquisa realizada por Rosa et al. (2009), os quais encontraram alta abundância de Sarcophagidae, Muscidae e Ulidiidae em ambiente de Cerrado sensu stricto em Uberlândia-MG, utilizando como isca carcaça suína. Estudo realizado em área de mata em Itumbiara-GO, utilizando como iscas rins de bovinos, vísceras de galinha, fezes humanas e peixe, apresentou padrão de famílias coletadas semelhante a este, com maior frequência da família Muscidae (MARCHIORI et al., 2003c). Em contrapartida, o padrão de famílias coletadas em outras regiões do Cerrado no Brasil apresentou-se diferente, como em Brasília, Distrito Federal, onde a família Calliphoridae foi a mais abundante com a utilização de carne bovina moída como isca (CASTRO et al., 2010).

Apesar da baixa frequência de indivíduos da família Fanniidae, sua presença pode ser explicada pelo hábito alimentar destas mocas. Elas são saprófagas e ocorrem em todos os tipos de matéria orgânica animal e vegetal em decomposição e também em fezes (COURI; CARVALHO, 2005). Entretanto, a presença da família Tabanidae pode ter sido acidental, uma vez que os machos da família alimentam-se de néctar, enquanto que as fêmeas da maioria das espécies são hematófagas (TURCATEL et al., 2007).
As iscas utilizadas no presente estudo não foram apropriadas para as famílias Micropezidae, Tephritidae e Sciaridae, pois os indivíduos destas famílias alimentamse de matéria vegetal, principalmente em decomposição (McALPINE, 1993). Entretanto, a presença dessas moscas pode ter sido influenciada pela a associação de alguns fatores, como local da coleta, disponibilidade de luz e alimentos (FRAGA; D'ALMEIDA, 2005).

Os sarcofagídeos estão distribuídos em regiões de clima tropical a temperado quente (PAPE, 1996; BARROS, et al., 2006), o que corrobora a presença destes indivíduos na região. A escolha das iscas foi apropriada para esta família, uma vez que suas larvas desenvolvem-se em matéria orgânica de origem animal em decomposição, preferencialmente em carcaças de vertebrados pequenos ou médios, podendo ocorrer também em certos grupos de invertebrados (BARROS et al., 2006).

As fêmeas do gênero Peckia utilizam preferencialmente fezes para realizar a larviposição (LOPES, 1973). Suas larvas desenvolvem-se em excrementos e em cadáveres de invertebrados e vertebrados, incluindo os humanos (JIRÓN et al., 1983; REEVES et al., 2000; BUENAVENTURA; PAPE, 2013). Possuem distribuição restrita à região neotropical; no entanto, há registro de espécies na parte sul das Regiões Neártica e Oceania (PAPE, 1996; PAPE; ANDERSSON, 2001; BUENAVENTURA; PAPE, 2013).

Mesmo que a maioria das espécies de Sarcophagidae esteja associada a ambientes antrópicos, algumas espécies ocorrem comumente em ambientes naturais, como a espécie Oxyvinia excisa (Lopes, 1950) que habita ambientes florestais. A presença desta espécie se deve à característica do habitat local, ao hábito alimentar coprófago, principalmente das fêmeas, e à procura de fezes para fazer a larviposição (LINHARES, 1981; D'ALMEIDA; ALMEIDA, 1998).

Um fato importante nesse estudo foi o registro inédito da espécie $P$. schildi no território brasileiro. Até então, havia registro desta espécie somente na Costa Rica (DODGE, 1963; CARVALHO, 2002). O fato do Cerrado ser pouco conhecido não permite, neste momento, descartar a hipótese de que a ausência de registros desta 
espécie nesta região seja devido aos poucos estudos realizados até então neste bioma sobre sua dipterofauna.

Várias espécies de Calliphoridae têm sido encontradas em ambientes antrópicos (rurais e urbanos) do Brasil, como no caso das espécies de Chrysomya Robineau-Desvoidy 1830 (BATISTA-DA-SILVA et al., 2010). Já $H$. segmentaria e Mesembrinella bicolor (Fabricius, 1805) tem sido encontradas associadas a ambientes naturais (GUIMARÃES, 1977; MELLO et al., 2007). Estas espécies apresentam distribuição Neotropical (MELLO, 1967; GUIMARÃES, 1977; DEAR, 1985; MELLO et al., 2007). Entretanto há poucas informações sobre a biologia das mesmas. Hemilucilia segmentaria é necrófaga, e suas larvas geralmente se alimentam de matéria em decomposição de animais e fezes (LINHARES, 1981; KOSMANN et al., 2011), enquanto as espécies de Mesembrinella (Giglio-Tos, 1893) estão associadas principalmente com a decomposição animal e vegetal (MELLO, 1967; GUIMARÃES, 1977; WOLFF, 2013).

Apesar de serem potenciais veiculadores de patógenos e causadores de mí́ases aos humanos e outros animais, as espécies de Chrysomya são importantes para os fragmentos do ponto de vista ecológico, por contribuírem na eliminação de carcaças, fezes e outros tipos de matéria orgânica em decomposição no local de estudo (CATTS; GOFF, 1992; CORRÊA et al., 2010).

Dentre as 29 espécies identificadas, Cyrtoneuropsis brunnea (Hough, 1900), Cyrtoneuropsis similata (Couri, 1982), O. excisa, P. schildi foram consideradas como potenciais indicadoras de ambiente florestal, uma vez que não há relatos publicados destas espécies em ambientes antrópicos. Estes dados mostram que mesmo alterados, alguns ambientes ainda apresentam espécies menos sensíveis às perturbações sofridas naquela área. Isto reforça a necessidade e importância da preservação destes ambientes.

Comparando os resultados apresentados neste estudo com os apresentados pelos trabalhos até então realizados no sul de Goiás (MARCHIORI et al., 2003a; 2003b; 2003c; 2007); observa-se que, com exceção de $C$. albiceps, C. megacephala, O. thornax, $P$. chrysostoma e Ravinia belforti, as demais espécies de Calliphoridae,
Muscidae e Sarcophagidae relatadas, não haviam sido registradas nos trabalhos anteriores. Isso se deve em grande parte aos poucos estudos com esta abordagem na região.

A presença da biodiversidade encontrada no presente estudo reforça a necessidade da preservação dessas áreas e aponta a importância de estudos continuados nestes ambientes para o melhor conhecimento dessa fauna. A realização de estudos adicionais utilizando também outros tipos de isca e amostragens em diferentes ambientes (antrópicos e naturais) poderá ampliar o conhecimento sobre esta dipterofauna e demonstrar que sua diversidade nesta região é maior que a encontrada até o momento. Além disso, essas informações permitirão a realização de estudos comparativos mais precisos sobre a influência das ações humanas sobre este grupo de insetos neste ambiente.

\section{Agradecimentos}

À pesquisadora Márcia Souto Couri (Departamento de Entomologia, Museu nacional, UFRJ) pela identificação de espécimes de Muscidae e ao Grupo de Pesquisa de Entomologia do ILES/ULBRA.

\section{Referências}

AZEVEDO, F. R.; MOURA, M. A. R.; ARRAIS, M. S. B.; NERE, D. R. Composição da entomofauna da Floresta Nacional do Araripe em diferentes vegetações e estações do ano. Revista Ceres, Viçosa, v. 58, n. 6, p. 740-748, 2011.

BARBOLA, I. F.; ANAZAWA, T. M.; GEMIM, B. S.; SILVA, G. J. T.; FERREIRA, T. P.; LIMA, B. N. S.; SILVA, M. A. A fragmentação de habitats e a diversidade de Hymenoptera (Insecta) em remanescentes florestais dos campos gerais do Paraná. In: CONGRESSO DE ECOLOGIA DO BRASIL, VIII, 2007, Caxambu. Anais... Caxambu: CEB, 2007. p. 1-2.

BARROS, R. M.; PENTEADO-DIAS, A. M.; PUJOL-LUZ, J. R. Registro de Peckia (Squamatodes) trivittata (Curran) (Diptera, Sarcophagidae) parasitada por Gnathopleura semirufa (Brullé) (Hymenoptera, Braconidae, Alysiinae) no cerrado de Brasília, DF. Revista Brasileira de Entomologia, São Paulo, v. 50, n. 3, p. 436438, 2006.

BRASIL. Biodiversidade brasileira: avaliação e identificação de áreas e ações prioritárias para a conservação, utilização sustentável e repartição dos benefícios da biodiversidade nos biomas brasileiros. 2002. Brasília: Ministério do Meio Ambiente. Disponível em: <http:www.biodiversidade.rs.gov.br/arquivos/ BiodiversidadeBrasileira_MMA.pdf >. Acesso em: 22 jan. 2014. 
BATISTA-DA-SILVA, J.A.; MOYA-BORJA, G. E. M.; QUEIROZ, M. M. de C. Ocorrência e sazonalidade de muscóides (Diptera, Calliphoridae) de importância sanitária no município de Itaboraí, RJ, Brasil. EntomoBrasilis, Vassouras, v. 3, n. 1, p. 16-21, 2010.

BUENAVENTURA, E.; PAPE, T. Revision of the genus Peckia Robineau-Desvoidy (Diptera: Sarcophagidae). Zootaxa, Auckland, v. 3622, n. 1, p. 1-87, 2013.

CASTRO, R. C. S.; DIAS, D. X.; SUJII, E. R.; QUEIROZ, P. R. Levantamento de dípteros de interesse forense em uma área de Cerrado em Brasília Universitas: Ciências da Saúde, Brasília, v. 8, n. 1, p. 1-16, 2010.

CARVALHO, C. J. B. Muscidae (Diptera) of the neotropical region: taxonomy. Curitiba: UFPR, 2002. $287 \mathrm{p}$.

CARVAlHO, C. J. B.; MELlO-PATIU, C. A. Keys to the adults of the most common forensic species of Diptera in South America. Revista Brasileira de Entomologia, Curitiba, v. 52, n. 3, p. 390406, 2008.

CARVAlHO, C. J. B.; MOURA, M. O.; RIBEIRO, P. B. Chave para adultos de dípteros (Muscidae, Fanniidae, Anthomyiidae) associados ao ambiente humano no Brasil. Revista Brasileira de Entomologia, Curitiba, v. 46, n. 2, p. 107-114, 2002.

CATTS, E. P.; GOFF, M. L. Forensic entomology in criminal investigations, departament of entomology. Annual Review of Entomology, Palo Alto, v. 37, n. 1, p. 253-272, 1992.

CORRÊA, E. C.; KOLLER, W. W.; BARROS, A. T. M. Abundância relativa e sazonalidade de espécies de Chrysomya (Diptera: Calliphoridae) no Pantanal Sul-Mato-Grossense, Brasil. Revista Brasileira de Parasitologia Veterinária, Jaboticabal, v. 19, n. 2, p. 85-88, 2010.

COURI, M. S; CARVALHO, C. J. B. Catálogo das espécies de Fanniidae do estado do Rio de Janeiro (Brasil). Biota Neotropica, Campinas, v. 5, n. 2, p. 223-231, 2005.

D’ALMEIDA, J. M.; ALMEIDA, J. R. Nichos tróficos em dípteros caliptrados, no Rio de Janeiro, RJ. Revista Brasileira de Biologia, São Carlos, v. 58, n. 4, p. 563-570, 1998.

DEAR, J. P. A revision of the new world Chrysomyini (Diptera: Calliphoridae). Revista Brasileira de Zoologia, Curitiba, v. 3, n. 3, p. 109-169, 1985.

DODGE, H. R. A New Philornis with coprophagous larva, and some related species (Diptera: Muscidae). Journal of the Kansas Entomological Society, Kansas, v. 36, n. 4, p. 239-247, 1963.

FRAGA, M. B.; D'ALMEIDA, J. M. Observações preliminares sobre a atratividade por diferentes cores em Calliphoridae (Diptera) Niterói, RJ, Brasil. Entomologia y Vectores, Rio de Janeiro, v. 12, n. 1, p. 141-147, 2005.

GUIMARÃES, J. H. A systematic revision of the Mesembrinellidae, stat. nov. (Diptera, Cyclorrhapha). Arquivos de Zoologia, São Paulo, v. 29, n. 1, p. 1-109, 1977.

GULlAN, P. J.; CRANSTON, P. S. Os insetos: um resumo de entomologia. São Paulo: Editora ROCA, 2008. 456 p.

JACKSON, H. B.; FAHRIG, L. Habitat loss and fragmentation. In: LEVIN, S. (Ed.). Encyclopedia of biodiversity. Waltham: Academic Press, 2013. 5504 p.

JIRÓN, L. F.; VARGAS, L. G.; VARGAS-ALVARADO, E. Four muscoid flies (Sarcophagidae and Muscidae) associated with human cadavers in Costa Rica. Brenesia, San Jose, v. 21, n. 21, p. 3-5, 1983.
KLINK, C. A; MACHADO, R. B. A conservação do Cerrado brasileiro. Megadiversidade, Belo Horizonte, v. 1, n. 1, p. 147$155,2005$.

KOSMANN, C.; MACEDO, M. P.; BARBOSA, T. A. F.; PUJOLLUZ, J. R. Chrysomya albiceps (Wiedemann) and Hemilucilia segmentaria (Fabricius) (Diptera, Calliphoridae) used to estimate the post-mortem interval in a forensic case in Minas Gerais, Brazil. Revista Brasileira de Entomologia, São Paulo, v. 55, n. 4, p. 621 623, 2011.

LINHARES, A. X. Synanthropy of Calliphoridae and Sarcophagidae (Diptera) in the city of Campinas, São Paulo, Brazil. Revista Brasileira de Entomologia, São Paulo, v. 25, n. 3, p. 189$215,1981$.

LOMÔNOCO, C.; ALMEIDA, J. R. de. Sazonalidade e uso de recursos para alimentação e oviposição de dípteros muscóides na restinga de Jacararepaguá, Rio de Janeiro, Brasil. Revista Brasileira de Entomologia, Curitiba, v. 39, n. 3, p. 883-890, 1995. LOPES, H. S. Collecting and rearing Sarcophagidae flies (Diptera) in Brazil during forty years. Anais da Academia Brasileira de Ciências, Rio de Janeiro, v. 45, n. 1, p. 279-291, 1973.

MARCHIORI, C. H.; CALDAS, E. R.; ALMEIDA, K. G. S.; LINHARES, A. X. Muscoid dipterous collected from cattle dung pats in pastures in Itumbiara, Goiás, Brazil. Arquivo Brasileiro de Medicina Veterinária e Zootecnia, Belo Horizonte, v. 55, n. 1, s/ paginação, 2003a.

MARCHIORI, C. H.; PEREIRA, L. A.; FILHO, O. M. S. Aphaereta sp. (Hymenoptera: Braconidae: Alysiinae) as a natural enemy to Peckia chrysostoma (Wiedemann) (Diptera: Sarcophagidae), in Brazil. Brazilian Journal of Biology, São Carlos, v. 63, n. 1, p. 169-172, 2003 b.

MARCHIORI, C. H.; PEREIRA, L. A.; FILHO, O. M. S.; RIBEIRO, L. C. S.; BORGES, V. R.; ARANTES, S. B. Microhimenópteros parasitóides de dípteros muscóides em diferentes substratos em área de mata de Itumbiara Goiás. Arquivo Brasileiro de Medicina Veterinária e Zootecnia, Belo Horizonte, v. 55, n. 4, p. 510-513, 2003c.

MARCHIORI, C. H.; SILVA-FILHO, O. M.; MILHOMEM, M. E. V.; LELES, A. S. Parasitóides de dípteros coletados em fezes de búfalos em uma propriedade rural em Itumbiara, Goiás. Arquivo Brasileiro de Medicina Veterinária e Zootecnia, Belo Horizonte, v. 59, n. 6, p. 1589-1592, 2007.

McAlPINE, J. F. Manual of Neartic Diptera. Ottawa: Biosystematics Research Centre Canada, 1987. 657 p.

McAlPINE, J. F. (Ed.). Manual of Nearctic Diptera. Ottawa: Biosystematics Research Centre Canada, 1993. 1581 p.

MClAughlin, Ó. B.; EMMERSON, M. C.; O’GORMAN, E. J. Chapter four - habitat isolation reduces the temporal stability of island ecosystems in the face of flood disturbance. Advances in Ecological Research, Orlando, v. 48, n. 3, p. 225-284, 2013.

MELO, A. S. O que ganhamos 'confundindo' riqueza de espécies e equabilidade em um índice de diversidade? Biota Neotropica, Campinas, v. 8, n. 3, p. 21-27, 2008.

MELLO, R. P. DE. Contribuição ao estudo dos Mesembrinellinae sul-americanos (Diptera, Calliphoridae). Studia Entomologica, Petrópolis, v. 10, n. 1, p. 1-80, 1967.

MELlO, R. S.; QUEIROZ, M. M. C.; AGUIAR-COELHO, V. M. Population fluctuations of calliphoridae species (Diptera, 
Calliphoridae) in the Biological Reserve of Tinguá. Iheringia, Série Zoologia, Porto Alegre, v. 97, n. 4, p. 1-5, 2007.

MORALES, M. N.; KÖHLER, A. Espécies de Syrphidae (Diptera) visitantes das flores de Eryngium horridum (Apiaceae) no Vale do Rio Pardo, RS, Brasil. Iheringia, Série Zoologia, Porto Alegre, v. 96, n. 1, p. 41-45, 2006.

MYERS, N.; MITTERMEIER, R. A.; MITTERMEIER, C. G.; DA FONSECA, G. A. B.; KENT, J. Biodiversity hotspots for conservation priorities. Nature, London, v. 403, n. 24, p. 853-858, 2000.

NEWMAN, B. J.; LADD, P.; BRUNDRETT M. C.; DIXON, K. W. Effects of habitat fragmentation on plant reproductive success and population viability at the landscape and habitat scale. Biological Conservation, Washington, v. 159, n. 1, 16-23, 2013.

OLIVEIRA, R. C.; FONSECA, A. R.; SILVA, C. G.; SIDNEY, L. A.; FERREIRA, C. P. S. Fauna de dípteros em uma área de cerrado no município de Divinópolis, estado de Minas Gerais. Revista Trópica: Ciências Agrárias e Biológicas, Chapadinha, v. 2, n. 2, p. 3-7, 2008.

PAPE, T. Catalogue of the Sarcophagidae of the world (Insecta: Diptera). Memoirs of Entomology, International, Gainsville, v. 8, n. 1, p. 1-558, 1996.

PAPE, T.; ANDERSSON, M. A new species of Peckia (Diptera: Sarcophagidae) from Costa Rica, with a note on P. pexata (Wulp). Insecta Mundi, Gainsville, v. 14, n. 4, p. 233-239, 2001.

RATTER, J. A.; RIBEIRO, J. F.; BRIDGEWATER, S. The Brazilian Cerrado vegetation and threats to its biodiversity. Annals of Botany, Oxford, v. 80, n. 3, p. 223-230, 1997.

REEVES, W. K.; PAPE, T.; ADLER, P. H. Biological notes on new world Sarcophagidae (Diptera). Studia Dipterologica, Müncheberg, v. 7, n. 2, p. 497-500, 2000.
ROSA, T. A.; BABATA, M. L. Y.; SOUZA, C. M.; SOUSA, D.; MELLO-PATIU, C. A.; MENDES, J. Dípteros de interesse forense em dois perfis de vegetação de Cerrado em Uberlândia, MG. Neotropical Entomology, Piracicaba, v. 38, n. 6, p. 859-866, 2009.

SOS MATA ATLÂNTICA; INPE - INSTITUTO NACIONAL DE PESQUISAS ESPACIAIS. Atlas dos remanescentes florestais da Mata Atlântica período de 2008-2010. São Paulo, 2011. Disponível em: <http: http://www.inpe.br/noticias/arquivos/pdf/ atlasrelatoriofinal.pdf $>$. Acesso em: 28 fev. 2014.

THOMANZINI, M. J.; THOMANZINI, A. P. B. W. Levantamento de insetos e análise entomofaunística em floresta, capoeira e pastagem no sudeste acreano. Rio Branco: EMBRAPA, 2002. 41 p. (Circular Técnica 35)

TURCATEL, M.; CARVAlHO, C. J. B; RAFAEL, J. A. Mutucas (Diptera: Tabanidae) do estado do Paraná, Brasil: chave de identificação pictórica para subfamílias, tribos e gêneros. Biota Neotropica, Campinas, v. 7, n. 2, p. 265-278, 2007.

VAIRO, K. P.; MELlO-PATIU, C. A.; CARVAlHO, C. J. B. Pictorial identification key for species of Sarcophagidae (Diptera) of potential forensic importance in southern Brazil. Revista Brasileira de Entomologia, Curitiba, v. 55, n. 3, p. 333-347, 2011. WINK, C.; GUEDES, J. V. C.; FAGUNDES, C. K.; ROVEDDER, A. P. Insetos edáficos como indicadores da qualidade ambiental. Revista de Ciências Agroveterinárias, Lages, v. 4, n. 1, p. 60-71, 2005.

WOLFF, M. A new species of Mesembrinella (Diptera: Calliphoridae: Mesembrinellinae) from Colombia. Revista Colombiana de Entomología, Bogotá, v. 39, n. 1, p. 120-124, 2013. 